\title{
$\Delta \pi N$ coupling constant in light cone QCD sum rules
}

\author{
A. Gokalp* and O. Yilmaz ${ }^{\dagger}$ \\ Physics Department, Middle East Technical University, \\ 06531 Ankara, Turkey
}

July 5,2021

\begin{abstract}
We employ the light cone QCD sum rules to calculate $\Delta \pi N$ coupling constant by studying the two point correlation function between the vacuum and the pion state. Our result is consistent with the traditional QCD sum rules calculations and it is in agreement with the experimental value.
\end{abstract}

*agokalp@metu.edu.tr

†oyilmaz@metu.edu.tr 


\section{Introduction}

The determination of hadronic parameters, in particular meson-baryon coupling constants, requires some information about the physics at large distances. Therefore one has to employ some specific nonperturbative method to obtain detailed predictions. Among the various nonperturbative methods, QCD sum rules [1] have proved to be very useful to extract the low-lying hadron masses and coupling constants. This method is a framework which connects hadronic parameters with QCD parameters. It is based on short-distance operator product expansion (OPE) in the deep Euclidean region of vacuum-vacuum correlation function in terms of quark and gluon condensates. Further progress has been achieved by an alternative method [2] known as the QCD sum rules on the light cone. The light cone QCD sum rules method is based on OPE on the light cone which is an expansion over the twist of the operators rather than the dimensions of the operators as in the traditional QCD sum rules approaches. In this expansion, the main contribution comes from the lowest twist operators. The matrix elements of nonlocal composite operators between meson and vacuum states define the meson wave functions. The light-cone QCD sum rules have been employed to study hadronic properties and these application can be found in [3]-[10] and references therein.

In this work we study the $p \pi^{+} \rightarrow \Delta^{++}$transition and we employ the light-cone QCD sum rules approach to calculate the $\Delta \pi N$ coupling $g_{\Delta \pi N}$. We note that this coupling constant was investigated by using the traditional QCD sum rules method in the soft pion limit [11]. In the spirit of the philosophy of the light cone QCD sum rules we study the time-ordered two-point correlation function of the interpolating field for $N$ and $\Delta$ with pion. We use a dispersion relation that relates the hadronic spectral density to the correlation function and thus write the correlator in terms of phenomenological hadronic spectrum. Since the interpolating fields are written in terms of quark fields, we also calculate this correlator directly from QCD on the light cone. We consider the structure $q_{\mu}$ and match these two descriptions. In order to calculate the coupling constant $g_{\Delta \pi N}$, we invoke the double Boorel transformation so that the excited states and the continuum contributions can be safely seperated out.

We consider the two-point correlation function with pion

$$
\Pi_{\mu}(p, q)=\int d^{4} x e^{i p x}<0\left|T\left\{\eta_{\mu}^{\Delta}(x) \bar{\eta}^{N}(0)\right\}\right| \pi^{+}(q)>
$$

with $p$ and $\eta_{\mu}^{\Delta}$ the four-momentum and the interpolating current of delta, $\eta^{N}$ the interpolating current of nucleon, and $q$ the four-momentum of pion. The interpolating currents for delta and nucleon [12] are 


$$
\begin{aligned}
\eta_{\mu}^{\Delta} & =\epsilon_{a b c}\left(u_{a}^{T} C \gamma_{\mu} u_{b}\right) u_{c} \\
\eta^{N} & =\epsilon_{a b c}\left(u_{a}^{T} C \gamma_{\mu} u_{b}\right) \gamma_{5} \gamma^{\mu} d_{c}
\end{aligned}
$$

where a,b,c are the color indices, $C=i \gamma_{2} \gamma_{0}$ is the charge conjugation matrix, $\mathrm{T}$ denotes transpose, and $u$ and $d$ are up and down quark fields respectively.

In order to construct the phenomenological side of the correlator we use the following Lagrangian density

$$
\mathcal{L}=g_{\Delta \pi N} \bar{N} \Delta_{\mu} \partial^{\mu} \pi
$$

which defines the $\Delta \pi N$ coupling constant $g_{\Delta \pi N}$ and where $N, \Delta_{\mu}$ and $\pi$ are the nucleon, delta and pion fields. Thus, at the phenomenological level the correlator can be saturated by delta and nucleon states as

$$
\Pi_{\mu}(p, q)=\frac{<0\left|\eta_{\mu}^{\Delta}\right| \Delta><\Delta|N \pi><N| \bar{\eta}^{N} \mid 0>}{\left(p^{2}-m_{\Delta}^{2}\right)\left(p^{\prime 2}-m_{N}^{2}\right)}+\ldots
$$

with the contributions from the higher states and the continuum denoted by dots. The overlapping amplitudes of the interpolating currents with delta and nucleon states are

$$
\begin{aligned}
& <N\left|\bar{\eta}^{N}\right| 0>=\lambda_{N} \bar{u}^{N}\left(p^{\prime}\right) \\
& <0\left|\eta_{\mu}^{\Delta}\right| \Delta>=\lambda_{\Delta} u_{\mu}^{\Delta}(p)
\end{aligned}
$$

where $u_{\mu}^{\Delta}$ is the Rarita-Schwinger spinor and the matrix element $\langle\Delta| N \pi>$ is given as

$$
<\Delta \mid N \pi>=-g_{\Delta \pi N} q^{\nu} \bar{u}_{\mu}^{\Delta}(p) u^{N}\left(p^{\prime}\right) \quad
$$

Substitution of Eqs. $(6,7,8)$ into Eq. (5) results in

$$
\begin{aligned}
& \Pi_{\mu}(p, q)=-\frac{\lambda_{N} \lambda_{\Delta} g_{\Delta \pi N}}{\left(p^{2}-m_{\Delta}^{2}\right)\left(p^{\prime 2}-m_{N}^{2}\right)} \quad\left[g_{\mu \nu}-\frac{1}{3} \gamma_{\mu} \gamma_{\nu}-\frac{2 p_{\mu} p_{\nu}}{3 m_{\Delta}^{2}}+\frac{\gamma_{\mu} p_{\nu}-\gamma_{\nu} p_{\mu}}{3 m_{\Delta}}\right] \\
& \times\left(\not p+m_{\Delta}\right) q^{\nu}\left(\not p^{\prime}+m_{N}\right)+\ldots
\end{aligned}
$$

We then consider the theoretical part of the correlator Eq. (1), for which we obtain

$$
\Pi_{\mu}(p, q)=\int d^{4} x e^{i p x}\left[-2\left(S\left(\gamma_{\nu} C\right) S^{T}\left(C \gamma_{\mu}\right) \mathcal{M}\left(\gamma^{\nu} \gamma_{5}\right)-\mathcal{M}\left(\gamma^{\nu} \gamma_{5}\right) \operatorname{tr}\left(\left(C \gamma_{\mu}\right) S\left(\gamma_{\nu} C\right) S^{T}\right)\right]\right.
$$

with

$$
\begin{aligned}
\mathcal{M}= & \gamma_{5}<0\left|\bar{d}_{a}(0) \gamma_{5} u_{a}(x)\right| \pi>-\gamma_{5} \gamma_{\lambda}<0\left|\bar{d}_{a}(0) \gamma_{5} \gamma^{\lambda} u_{a}(x)\right| \pi> \\
& +\frac{1}{2} \sigma_{\alpha \beta}<0\left|\bar{d}_{a}(0) \sigma^{\alpha \beta} u_{a}(x)\right| \pi>
\end{aligned}
$$


The full light quark propagator in Eq. (10) with both perturbative term and contribution from vacuum fields is given as

$$
\begin{aligned}
i S(x)=\quad & i \frac{\not x}{2 \pi^{2} x^{4}}-\frac{<\bar{q} q>}{12}-\frac{x^{2}}{192}<\bar{q} g_{s} \sigma \cdot G q> \\
& -i \frac{g_{s}}{16 \pi^{2}} \int_{0}^{1} d u\left\{\frac{\not x}{x^{2}} \sigma \cdot G(u x)-4 i u \frac{x_{\mu}}{x^{2}} G^{\mu \nu}(u x) \gamma_{\nu}\right\}+\ldots
\end{aligned}
$$

The matrix elements of the nonlocal operators between the vacuum and pion state defines the two particle pion wave functions, and up to twist four the Dirac components of these wave functions can be written as 13

$$
\begin{aligned}
<0\left|\bar{d}(0) \gamma_{\mu} \gamma_{5} u(x)\right| \pi^{+}>= & i f_{\pi} q_{\mu} \int_{0}^{1} d u e^{-i q u x}\left(\varphi_{\pi}(u)+x^{2} g_{1}(u)\right) \\
& +f_{\pi}\left(x_{\mu}-\frac{x^{2} q_{\mu}}{q^{2}}\right) \int_{0}^{1} d u e^{-i q u x} g_{2}(u) \\
<0\left|\bar{d}(0) i \gamma_{5} u(x)\right| \pi^{+}>= & \frac{f_{\pi} m_{\pi}^{2}}{m_{u}+m_{d}} \int_{0}^{1} d u e^{-i q u x} \varphi_{P}(u), \\
<0\left|\bar{d}(0) \sigma^{\mu \nu} \gamma_{5} u(x)\right| \pi^{+}>= & \left(q_{\mu} x_{\nu}-q_{\nu} x_{\mu}\right) \frac{i f_{\pi} m_{\pi}^{2}}{6\left(m_{u}+m_{d}\right)} \int_{0}^{1} d u e^{-i q u x} \varphi_{\sigma}(u) .
\end{aligned}
$$

We further define

$$
G_{2}(u)=-\int_{0}^{u} g_{2}(v) d v, \quad G_{2}(1)=G_{2}(0)=0
$$

and

$$
g_{3}(u)=g_{1}(u)+G_{2}(u) \quad
$$

From the theqretical part of the correlator given in Eq. (10), we consider the structure $q_{\mu}$, and for this structure after Fourier transformation over $x$ and double Borel transformations with respect to variables $p_{1}^{2}=p^{2}$ and $p_{2}^{2}=(p-q)^{2}$ we finally obtain

$$
\begin{aligned}
\Pi^{\text {theor. }}= & f_{\pi} \mu_{\pi}<\bar{q} q>\left[2 M^{2} f_{0}\left(s_{0} / M^{2}\right)-\frac{1}{2} m_{0}^{2}\right] \varphi_{P}\left(u_{0}\right) u_{0} \\
& +\frac{f_{\pi}}{\pi^{2}}\left[-\frac{1}{2} M^{6} f_{2}\left(s_{0} / M^{2}\right)+\frac{1}{24} g_{s}^{2}<G^{2}>M^{2} f_{0}\left(s_{0} / M^{2}\right)\right] \varphi_{\pi}\left(u_{0}\right) \\
& +\frac{f_{\pi}}{\pi^{2}}\left[-\frac{1}{6} M^{6} f_{2}\left(s_{0} / M^{2}\right)-\frac{1}{24} g_{s}^{2}<G^{2}>M^{2} f_{0}\left(s_{0} / M^{2}\right)\right] u_{0} \varphi_{\pi}^{\prime}\left(u_{0}\right) \\
& +\frac{f_{\pi}}{\pi^{2}} \frac{1}{6} g_{s}^{2}<G^{2}>g_{2}\left(u_{0}\right) \frac{f_{\pi}}{\pi^{2}} f_{1}\left(s_{0} / M^{2}\right) g_{3}\left(u_{0}\right) \\
& +\frac{f_{\pi}}{\pi^{2}}\left[2 M^{4} f_{1}\left(s_{0} / M^{2}\right)+\frac{1}{6} g_{s}^{2}<G^{2}>\right] u_{0} g_{3}^{\prime}\left(u_{0}\right) \\
& +\frac{1}{18} f_{\pi} \mu_{\pi}<\bar{q} q>\left[-8 M^{2} f_{0}\left(s_{0} / M^{2}\right) \varphi_{\sigma}\left(u_{0}\right)+\left(-4 M^{2} f_{0}\left(s_{0} / M^{2}\right)+m_{0}^{2}\right) u_{0} \varphi_{\sigma}^{\prime}\left(u_{0}\right)\right]
\end{aligned}
$$


where the function

$$
f_{n}\left(s_{0} / M^{2}\right)=1-e^{-s_{0} / M^{2}} \sum_{k=0}^{n} \frac{\left(s_{0} / M^{2}\right)^{k}}{k !}
$$

is the factor used to subtract the continuum, which is modeled by the dispersion integral in the region $s_{1}, s_{2} \geq s_{0}, s_{0}$ being the continuum threshold, $\mu_{\pi}=\frac{m_{\pi}^{2}}{m_{u}+m_{d}}$, and

$$
u_{0}=\frac{M_{1}^{2}}{M_{1}^{2}+M_{2}^{2}}, \quad M^{2}=\frac{M_{1}^{2} M_{2}^{2}}{M_{1}^{2}+M_{2}^{2}}
$$

with $M_{1}^{2}$ and $M_{2}^{2}$ are the Borel parameters, and $\varphi^{\prime}\left(u_{0}\right)=\left.\frac{d \varphi}{d u}\right|_{u_{0}}$.

Performing double Borel transformation over the variables $p_{1}^{2}=p^{2}$ and $p_{2}^{2}=(p-q)^{2}$ on the phenomenological part in Eq. (9), and then equating the result obtained for the Lorentz structure $g_{\mu \nu}$ part to that theoretical result given in Eq. (18) we finally obtain the sum rule for the coupling constant $g_{\Delta \pi N}$

$$
g_{\Delta \pi N} \lambda_{N} \lambda_{\Delta}=\frac{2}{\left(m_{N}+m_{\Delta}\right)^{2}} e^{\frac{m_{\Delta}^{2}}{M_{1}^{2}}} e^{\frac{m_{N}^{2}}{M_{2}^{2}}} \Pi^{\text {theor. }} .
$$

We note that this sum rule depends on the value of the wave functions at a specific point, which are much better known than the whole wave functions [14].

\section{Numerical results and discussion}

The various parameters we adopt are $m_{0}^{2}=0.8 \mathrm{GeV}^{2},<g_{s}^{2} G^{2}>=0.474 \mathrm{GeV}^{4}, f_{\pi}=0.132 \mathrm{GeV}$, $\mu_{\pi}=1.65 \mathrm{GeV},<\bar{q} q>=-(0.225 \mathrm{GeV})^{3}, s_{0}=3 \mathrm{GeV}^{2}$. In our calculation of the theoretical part we use the two particle pion wave functions based on the QCD sum rule approach given in [14] as

$$
\begin{aligned}
\varphi_{\pi}(u, \mu)= & 6 u \bar{u}\left[1+a_{2}(\mu) C_{2}^{3 / 2}(2 u-1)+a_{4} C_{4}^{3 / 2}(2 u-1)\right], \\
\varphi_{\sigma}(u, \mu)= & 6 u \bar{u}\left[1+C_{2} \frac{3}{2}\left[5(u-\bar{u})^{2}-1\right]+C_{4} \frac{15}{8}\left[21(u-\bar{u})^{4}-14(u-\bar{u})^{2}+1\right]\right], \\
\varphi_{P}(u, \mu)= & 1+B_{2} \frac{1}{2}\left[3(u-\bar{u})^{2}-1\right]+B_{4} \frac{1}{8}\left[35(u-\bar{u})^{4}-30(u-\bar{u})^{2}+3\right], \\
g_{1}(u, \mu)= & \frac{5}{2} \delta^{2}(\mu) u^{2} \bar{u}^{2}+\frac{1}{2} \epsilon(\mu)\left[u \bar{u}(2+13 u \bar{u})+10 u^{3} \ln u\left(2-3 u+\frac{6}{5} u^{2}\right)\right. \\
& \left.+10 \bar{u}^{3} \ln \bar{u}\left(2-3 \bar{u}+\frac{6}{5} \bar{u}^{2}\right)\right], \\
g_{2}(u, \mu)= & \frac{10}{3} \delta^{2} u \bar{u}(u \bar{u}) \\
G_{2}(u, \mu)= & \frac{5}{3} \delta^{2} u^{2} \bar{u}^{2},
\end{aligned}
$$


where $\bar{u}=1-u, C_{2}^{3 / 2}$ and $C_{4}^{3 / 2}$ are the Gegenbauer polynomials defined as

$$
\begin{aligned}
C_{2}^{3 / 2}(2 u-1) & =\frac{3}{2}\left[5(2 u-1)^{2}+1\right], \\
C_{4}^{3 / 2}(2 u-1) & =\frac{15}{8}\left[21(2 u-1)^{4}-14(2 u-1)^{2}+1\right],
\end{aligned}
$$

and $a_{2}=2 / 3, a_{4}=0.43$ corresponding to the normalization point $\mu=0.5 \mathrm{GeV}$. The remaining parameters are taken from the QCD sum rule estimates of Ref. [15 as $\delta^{2}(\mu=1 \mathrm{GeV})=0.2$, and $\epsilon(\mu=1 \mathrm{GeV})=0.5$ and from those of Refs.[3], 13] as $B_{2}=0.48, B_{4}=1.51, C_{2}=0.10$, $C_{4}=0.07$. Since the mass difference of $N$ and $\Delta$ is not very significant, we let $M_{1}^{2}=M_{2}^{2}=2 M^{2}$ from which it follows that $u_{0}=1 / 2$.

We study the dependence of the sum rule of Eq. (19) on the continuum threshold $s_{0}$ and on the Borel parameter $M^{2}$. We find that the sum rule is very stable with resonable variations of $s_{0}$ and $M^{2}$ as can be seen in Fig. 1. In order to determine the value of the coupling constant $g_{\Delta \pi N}$ from the sum rule Eq. (19), the residues $\lambda_{\Delta}$ and $\lambda_{N}$ of the hadronic currents are needed. We use the following values that are obtained from the corresponding sum rules for $\Delta$ and $N$ which are 11]

$$
\begin{aligned}
\left|\lambda_{N}\right|^{2} 2(2 \pi)^{4} e^{-\frac{m_{N}^{2}}{M^{2}}} & =M^{6} f_{2}\left(s_{0}^{N} / M^{2}\right)+b M^{2} f_{0}\left(s_{0}^{N} / M^{2}\right)+\frac{4}{3} a^{2} \\
\left|\lambda_{\Delta}\right|^{2} 5(2 \pi)^{4} e^{-\frac{m_{\Delta}^{2}}{M^{2}}} & =M^{6} f_{2}\left(s_{0}^{\Delta} / M^{2}\right)-\frac{25}{18} b M^{2} f_{0}\left(s_{0}^{\Delta} / M^{2}\right)+\frac{20}{3} a^{2}
\end{aligned}
$$

where

$$
\begin{aligned}
a & =-2 \pi^{2}<\bar{q} q>=0.5 \mathrm{GeV}^{3}, \\
b & =\frac{\alpha_{s}}{\pi}<G^{2}>=0.12 \mathrm{GeV}^{4}, \quad \text { and } \\
s_{0}^{\Delta} & =\left(m_{\Delta}+0.5\right)^{2}, \quad s_{0}^{N}=\left(m_{N}+0.5\right)^{2} .
\end{aligned}
$$

We substitute these values of the residues into the sum rule in Eq. (19), and further study the dependence of $g_{\Delta \pi N}$ on the continuum threshold $s_{0}$ and on the Borel parameter $M^{2}$. This dependence is shown in Fig. 2, examination of which indicates that it is quite stable with resonable variations of $s_{0}$ and $M^{2}$. We thus obtain the coupling constant $g_{\Delta \pi N}$ as

$$
g_{\Delta \pi N}=(14.5 \pm 1.5) \mathrm{GeV}^{-1}
$$

The uncertainty we included is due to the variation of the continuum threshold and the Borel parameter. 
Finally, we consider the Lagrangian density in Eq. (4) and obtain the expression for the decay width as

$$
\Gamma\left(\Delta^{++} \rightarrow \pi^{+} p\right)=\frac{1}{6} \frac{g_{\Delta \pi N}^{2}}{4 \pi} \frac{\left(m_{N}+m_{\Delta}\right)^{2}-m_{\pi}^{2}}{m_{\Delta}^{2}} p_{\pi}^{3}
$$

where the pion momentum $p_{\pi}$ is

$$
p_{\pi}=\sqrt{\left(\frac{m_{\pi}^{2}+m_{\Delta}^{2}-m_{p}^{2}}{2 m_{\Delta}}\right)^{2}-m_{\pi}^{2}} .
$$

We use the experimental result [16] for the decay width and obtain the coupling constant as

$$
g_{\Delta \pi N}=(15.2 \pm 0.1) \quad G e V^{-1}
$$

which indicates that our value in Eq. (24) is in satisfactory agreement with the experimental result.

In summary, we calculated $\Delta \pi N$ coupling constant $g_{\Delta \pi N}$ using light-cone QCD sum rules. Our result is consistent with the results obtained using traditional QCD sum rules [11] and it is in good agreement with the value of the coupling constant deduced from the experimental decay rate of $\Delta^{++}$baryon.

\section{Acknowledgment}

We thank to T. M. Aliev for suggesting us this problem and his guidance during the course of our work. 


\section{References}

[1] M. A. Shifman, A. I. Vainshtein, and V. I. Zakharov, Nucl. Phys. B147 (1979) 385.

[2] V. L. Chernyok and A. R. Zhitnitsky, JETP Lett. 25 (1977) 510;

A. V. Efremov and A. V. Radyushkin, Phys. Lett. B94 (1980) 245.

G. P. Lepage and S. J. Brodsky, Phys. Lett. B87 (1979) 359.

[3] V. L. Chernyak and A. R. Zhitnitsky, Phys. Rep. 112 (1984) 173.

[4] V. M. Braun, In proc. "Rostock 1997, Progress in heavy quark physics" 105-118, 1997, prep. hep-ph/9801222 (1998)

[5] I. I. Balitskii, V. M. Braun, A. V. Kolesnichenko, Nucl. Phys. B312 (1989) 509;

[6] V. M. Braun and I. E. Filyanov, Z. Phys. C48 (1990) 238;

[7] V. L. Chernyak and A. R. Zhitnitsky, Nucl. Phys. B345 (1990) 137.

[8] P. Ball, V. M. Braun, Phys. Rev. D55 (1997) 5561;

[9] P. Ball, V. M. Braun, Phys. Rev. D58 (1998) 094016;

[10] T. M. Aliev, M. Savci, A. Özpineci, Phys. Rev. D56 (1997) 4260;

[11] L. J. Reinders, A. Rubinstein and S. Yazaki, Phys. Rep. 127 (1985) 1.

[12] B. L. Ioffe, Nucl Phys. B188 (1981) 317; Errata, Nucl. Phys. B191 (1981) 591.

[13] V. M. Braun and I. E. Filyanov, Z. Phys. C44 (1989) 157;

[14] V. M. Belyaev, V. M. Braun, A. Khodjamirian and R. Rückel, Phys. Rev. D51 (1995) 6177 ;

[15] V. A. Novikov, M. A. Shifman, A. I. Vainshtein, and V. I. Zakharov, Nucl. Phys. B237 (1984) 525.

[16] C. Caso et al., Review of Particle Properties, Euro. Phys. J. C3 (1998) 1. 


\section{Figure Captions:}

Figure 1: The dependence of $g_{\Delta \pi N} \lambda_{\Delta} \Lambda_{N}$ on the Borel parameter $M^{2}$ and on the continuum threshold $s_{0}$.

Figure 2: The sum rule for $g_{\Delta \pi N}$ as a function of the Borel parameter $M^{2}$ and the continuum threshold $s_{0}$. 


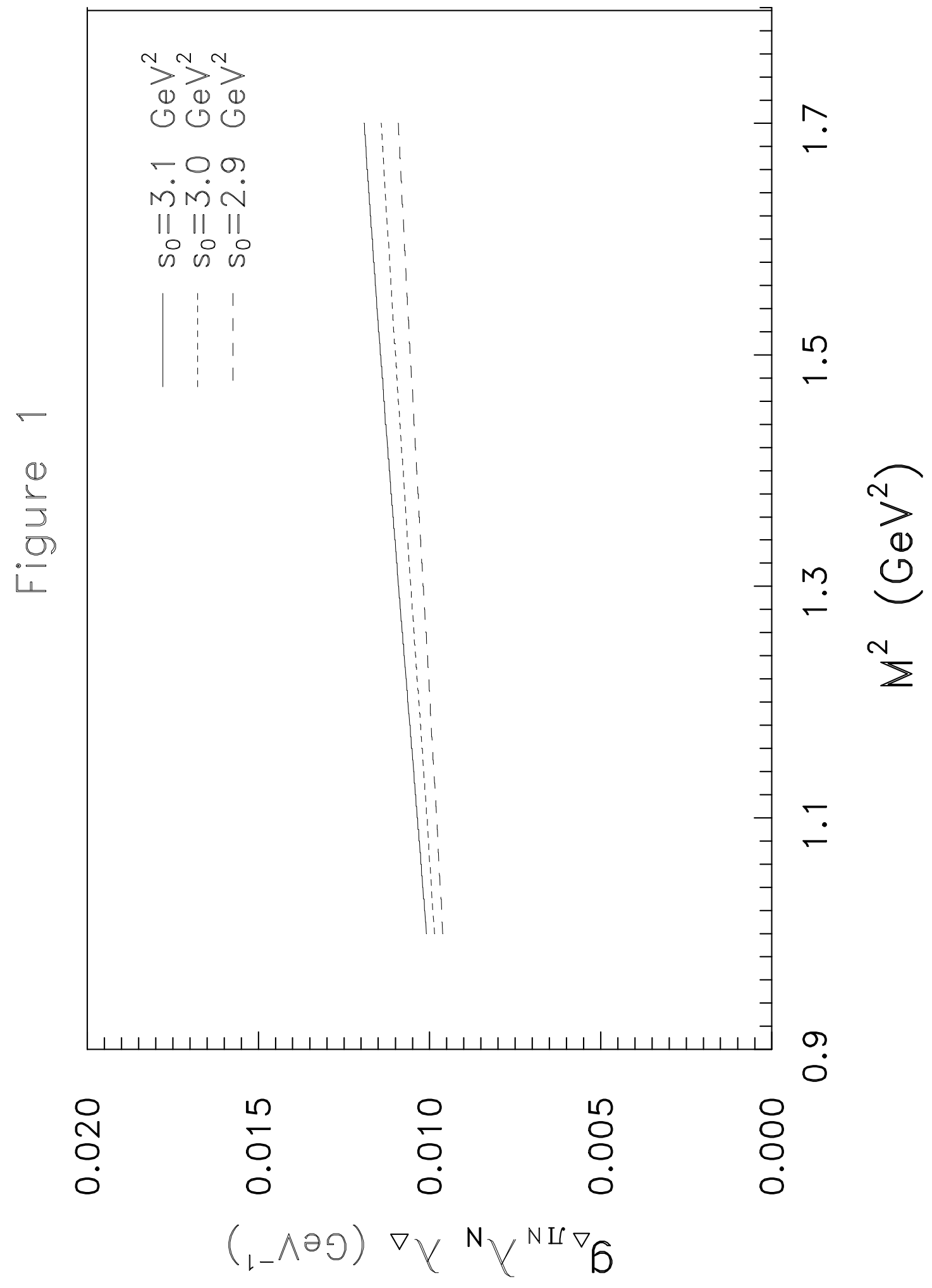




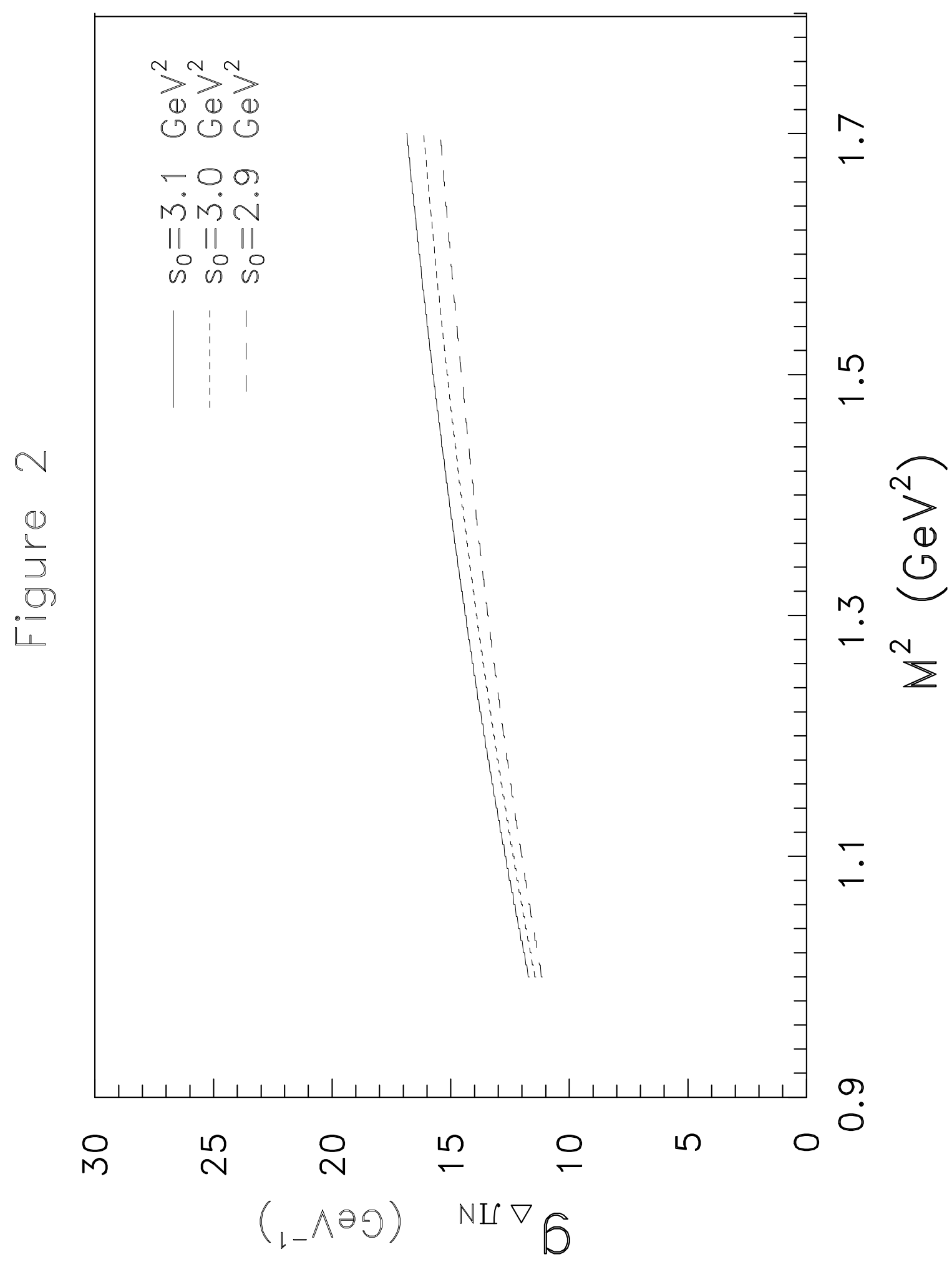

